\title{
Index of Roots (Syllabes Radicales)
}

the figures not preceded by a " $p$ " are the table numbers

les chiffres sans la lettre « $p$ s indiquent les nos des tables

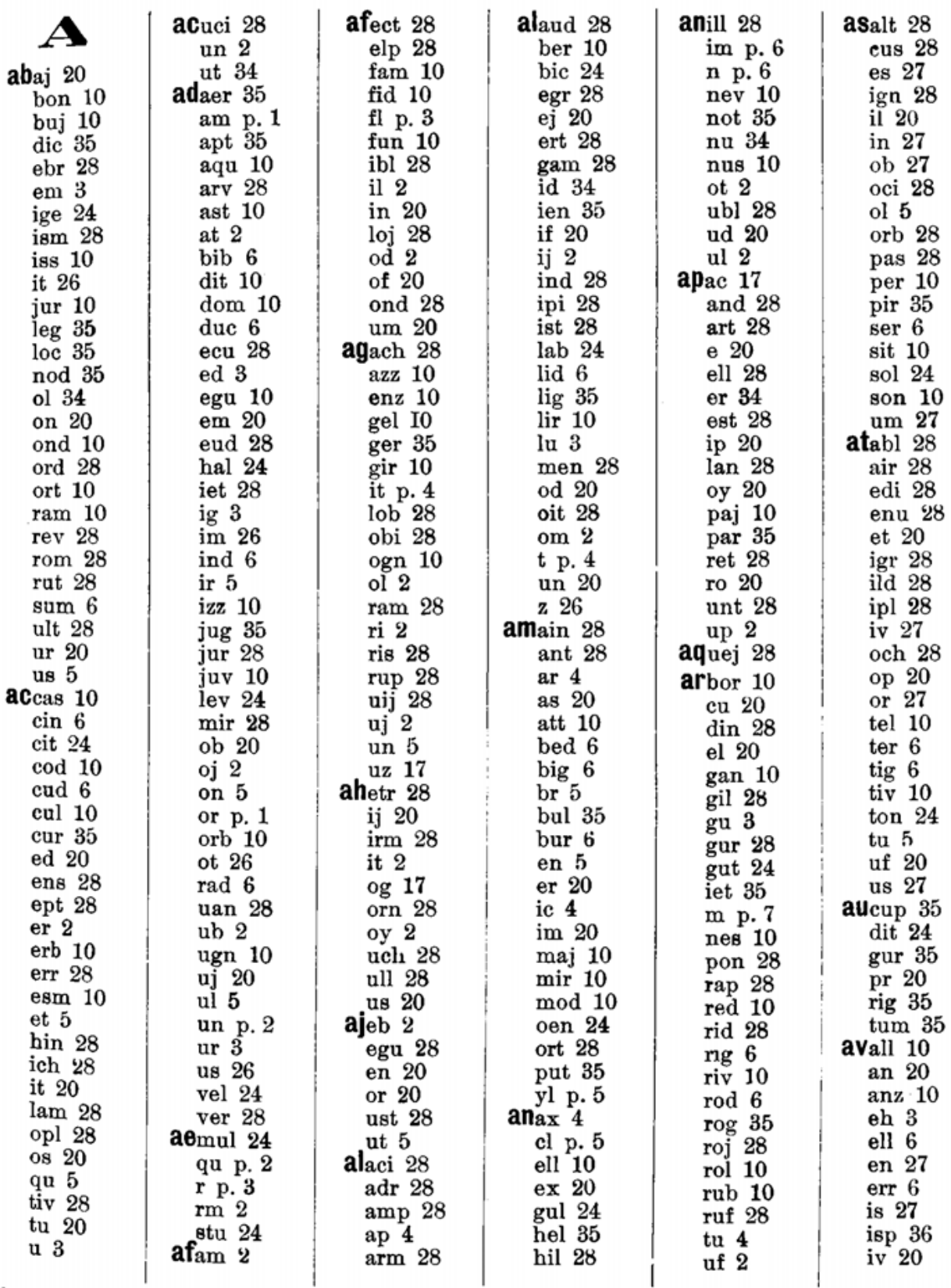

the first letter of a termination is always a vowel - la 1ere lettre d'une terminuison est toujours une voyelle 


\begin{tabular}{|c|c|c|c|c|c|}
\hline $\begin{array}{l}\text { bagn } 5 \\
\text { j } 26 \\
\text { jul } 10 \\
\text { ld } 20 \\
\text { li } 27 \\
\text { lte } 24 \\
\text { raj } 36 \\
\text { rd } 26 \\
\text { rlo } 36 \\
\text { rr } 5 \\
\text { si p. } 8 \\
\text { toj } 36 \\
\text { ttu } 6 \\
\text { ub } 4 \\
\text { bebd } 20 \\
\text { f } 20 \\
\text { ff } 26 \\
\text { ix } 20 \\
\text { Id } 27 \\
\text { ll } 4 \\
\text { nd } 13 \\
\text { tun } 36 \\
\text { biad } 13 \\
\text { b } 3 \\
\text { ff } 13 \\
\text { n } 27 \\
\text { ond } 10 \\
\text { rl } 20 \\
\text { tum } 10 \\
\text { ul } 26 \\
\text { zm } 20 \\
\text { blasm } 36 \\
\text { ind } 10 \\
\text { boch } 20 \\
\text { let } 36 \\
\text { ll } 13 \\
\text { nd } 27 \\
\text { rd } 13 \\
\text { rn } 27 \\
\text { rr } 20 \\
\text { sl } 20 \\
\text { ved } 36 \\
\text { y } 20 \\
\text { zz } 13\end{array}$ & $\begin{array}{l}\text { bram } 26 \\
\text { as } 20 \\
\text { av } 13 \\
\text { evi } 35 \\
\text { id } 27 \\
\text { ind } 36 \\
\text { om } 27 \\
\text { ot } 20 \\
\text { um } 20 \\
\text { un } 34 \\
\text { buh } 21 \\
\text { lin } 10 \\
\text { ll } 21 \\
\text { ril } 36 \\
\text { rl } 13 \\
\text { rx } 21 \\
\text { s } 26 \\
\text { sc } 17 \\
\text { ss } 13 \\
\text { tt } 13 \\
\text { C } \\
\text { cabs } 27 \\
\text { c p. } 8 \\
\text { ed } 3 \\
\text { el p. } 9 \\
\text { lce } 35 \\
\text { lig } 35 \\
\text { ll } 21 \\
\text { lm } 13 \\
\text { lon } 36 \\
\text { lv } 27 \\
\text { mbi } 36 \\
\text { mel } 36 \\
\text { mer } 24 \\
\text { mod } 36 \\
\text { mps } 24 \\
\text { nd } 21 \\
\text { ns } 13 \\
\text { nt p. } 9 \\
\text { pon } 10 \\
\text { pp } 13 \\
\text { pri } 24 \\
\text { pt } 21 \\
\text { pul } 24 \\
\text { rat } 10 \\
\text { rd } 27 \\
\text { rem } 36 \\
\text { ren } 10 \\
\text { rin } 24 \\
\text { rp } 34 \\
\text { str } 35 \\
\text { tt } 13 \\
\text { us } 21 \\
\text { ut } 26 \\
\text { vil } 36 \\
\text { z } 17 \\
\text { zz } 13 \\
\text { and }\end{array}$ & $\begin{array}{l}\text { ceb } 27 \\
\text { cin } 36 \\
\text { dr } 13 \\
\text { ff } 13 \\
\text { ler } 35 \\
\text { nn } 13 \\
\text { nsu } 10 \\
\text { pp } 26 \\
\text { rc } 17 \\
\text { rnu } 24 \\
\text { rr } 26 \\
\text { rt } 4 \\
\text { st } 34 \\
\text { chaf } 21 \\
\text { ant } 36 \\
\text { arp } 36 \\
\text { as } 27 \\
\text { et } 13 \\
\text { iod } 10 \\
\text { ipr } 36 \\
\text { om } 21 \\
\text { or } 27 \\
\text { orr } 36 \\
\text { um } 27 \\
\text { up } 21 \\
\text { urn } 36 \\
\text { cib } 26 \\
\text { cl } 21 \\
\text { cur } 10 \\
\text { fr } 13 \\
\text { gn } 23 \\
\text { ll } 3 \\
\text { m } 23 \\
\text { ng } 3 \\
\text { nt } 21 \\
\text { t p. } 10 \\
\text { uff } 10 \\
\text { urm } 10 \\
\text { v } 34 \\
\text { clam } 23 \\
\text { ar p. } 10 \\
\text { ass } 10 \\
\text { av } 21 \\
\text { ep } 3 \\
\text { in } 4 \\
\text { ipe } 35 \\
\text { is } 21 \\
\text { od } 3 \\
\text { coact } 24 \\
\text { add } 6 \\
\text { al } 3 \\
\text { bij } 36 \\
\text { br } 21 \\
\text { ed } 21 \\
\text { em } 3 \\
\text { en p. } 11 \\
\text { ept } 35 \\
\text { git } 36 \\
\text { hob } 36\end{array}$ & $\begin{array}{l}\text { c0ir p. } 11 \\
\text { ll } 26 \\
\text { lm } 22 \\
\text { lor } 35 \\
\text { lp } 23 \\
\text { ltr } 10 \\
\text { mb } 22 \\
\text { med } 6 \\
\text { mis } 36 \\
\text { mit } 35 \\
\text { mme } 35 \\
\text { mod } 10 \\
\text { mp } 34 \\
\text { mpr } 10 \\
\text { mun } 10 \\
\text { nd } 34 \\
\text { nfl } 35 \\
\text { nlo } 36 \\
\text { nsu } 6 \\
\text { ort } 10 \\
\text { pel } 36 \\
\text { pin } 36 \\
\text { qu } 3 \\
\text { rch } 36 \\
\text { rl } 22 \\
\text { rn } 23 \\
\text { ron } 35 \\
\text { rru } 6 \\
\text { rt } 27 \\
\text { vid } 10 \\
\text { vr } 34 \\
\text { yt } 22 \\
\text { crax p. } 12 \\
\text { em p. } 12 \\
\text { ep } 23 \\
\text { ib } 22 \\
\text { ibr } 35 \\
\text { in } 27 \\
\text { ism } 36 \\
\text { isp } 24 \\
\text { ost } 10 \\
\text { uci } 35 \\
\text { ust } 35 \\
\text { uz } 17 \\
\text { cuaj } 22 \\
\text { art } 36 \\
\text { b } 22 \\
\text { ch } 27 \\
\text { cin } 10 \\
\text { di } 27 \\
\text { est } 36 \\
\text { id } 22 \\
\text { it } 23 \\
\text { ll } 23 \\
\text { lp p. } 13 \\
\text { nct } 24 \\
\text { ne p. } 13 \\
\text { r } 26 \\
\text { rr 23 } \\
\text { rr }\end{array}$ & $\begin{array}{l}\text { Curs } 22 \\
\text { rv p. } 14 \\
\text { sc } 34 \\
\\
\text { D } \\
\text { dabi } 22 \\
\text { div } 36 \\
\text { m } 26 \\
\text { mn p. } 14 \\
\text { n } 22 \\
\text { nn } 23 \\
\text { pin } 24 \\
\text { t p. } 15 \\
\text { dealb } 35 \\
\text { am p. } 15 \\
\text { aur } 35 \\
\text { can } 6 \\
\text { cid } 6 \\
\text { cim } 10 \\
\text { cor } 10 \\
\text { dom } 24 \\
\text { flu } 6 \\
\text { gn } 23 \\
\text { gul } 24 \\
\text { gun } 6 \\
\text { hes } 36 \\
\text { j } 22 \\
\text { lav } 24 \\
\text { lax } 36 \\
\text { lib } 10 \\
\text { lig } 35 \\
\text { lin } 6 \\
\text { met } 9 \\
\text { mor } 36 \\
\text { mud } 36 \\
\text { neg } 9 \\
\text { nod } 10 \\
\text { not } 9 \\
\text { ns p. } 16 \\
\text { occ } 24 \\
\text { opt } 24 \\
\text { pil } 9 \\
\text { pon } 6 \\
\text { pos } 36 \\
\text { ps } 3 \\
\text { pur } 10 \\
\text { put } 9 \\
\text { puv } 6 \\
\text { rad } 6 \\
\text { riv } 9 \\
\text { rog } 9 \\
\text { ru } 3 \\
\text { sal } 10 \\
\text { sec } 24 \\
\text { sin } 10 \\
\text { soj } 36 \\
\text { sol } 9 \\
\text { and }\end{array}$ & $\begin{array}{l}\text { desud } 9 \\
\text { sug } 6 \\
\text { sun } 36 \\
\text { teg } 6 \\
\text { ton } 10 \\
\text { tt } 26 \\
\text { ung } 6 \\
\text { van } 36 \\
\text { ved } 36 \\
\text { vel } 24 \\
\text { vis } 36 \\
\text { vit } 9 \\
\text { vor } 9 \\
\text { zm } 22 \\
\text { dibuj } 36 \\
\text { ct p. } 16 \\
\text { fam } 36 \\
\text { ffl } 9 \\
\text { fil } 10 \\
\text { gn p. } 17 \\
\text { jug } 9 \\
\text { lat } 9 \\
\text { lu } 3 \\
\text { moj } 10 \\
\text { not } 10 \\
\text { nud } 10 \\
\text { pan } 10 \\
\text { pel } 10 \\
\text { put } 10 \\
\text { ren } 10 \\
\text { rim } 6 \\
\text { rob } 10 \\
\text { rup } 10 \\
\text { sat } 10 \\
\text { sc } 3 \\
\text { sen } 36 \\
\text { sip } 36 \\
\text { sir } 10 \\
\text { sus } 10 \\
\text { tt } 23 \\
\text { vex } 24 \\
\text { vid } 6 \\
\text { vin } 10 \\
\text { vor } 10 \\
\text { dobl } 26 \\
\text { l } 27 \\
\text { le } 34 \\
\text { men } 36 \\
\text { n p. } 17 \\
\text { s } 26 \\
\text { drag } 17 \\
\text { ans } 36 \\
\text { iz } 17 \\
\text { ell } 10 \\
\text { p } 22 \\
\text { pl } 4 \\
\text { r p. } 18\end{array}$ \\
\hline
\end{tabular}




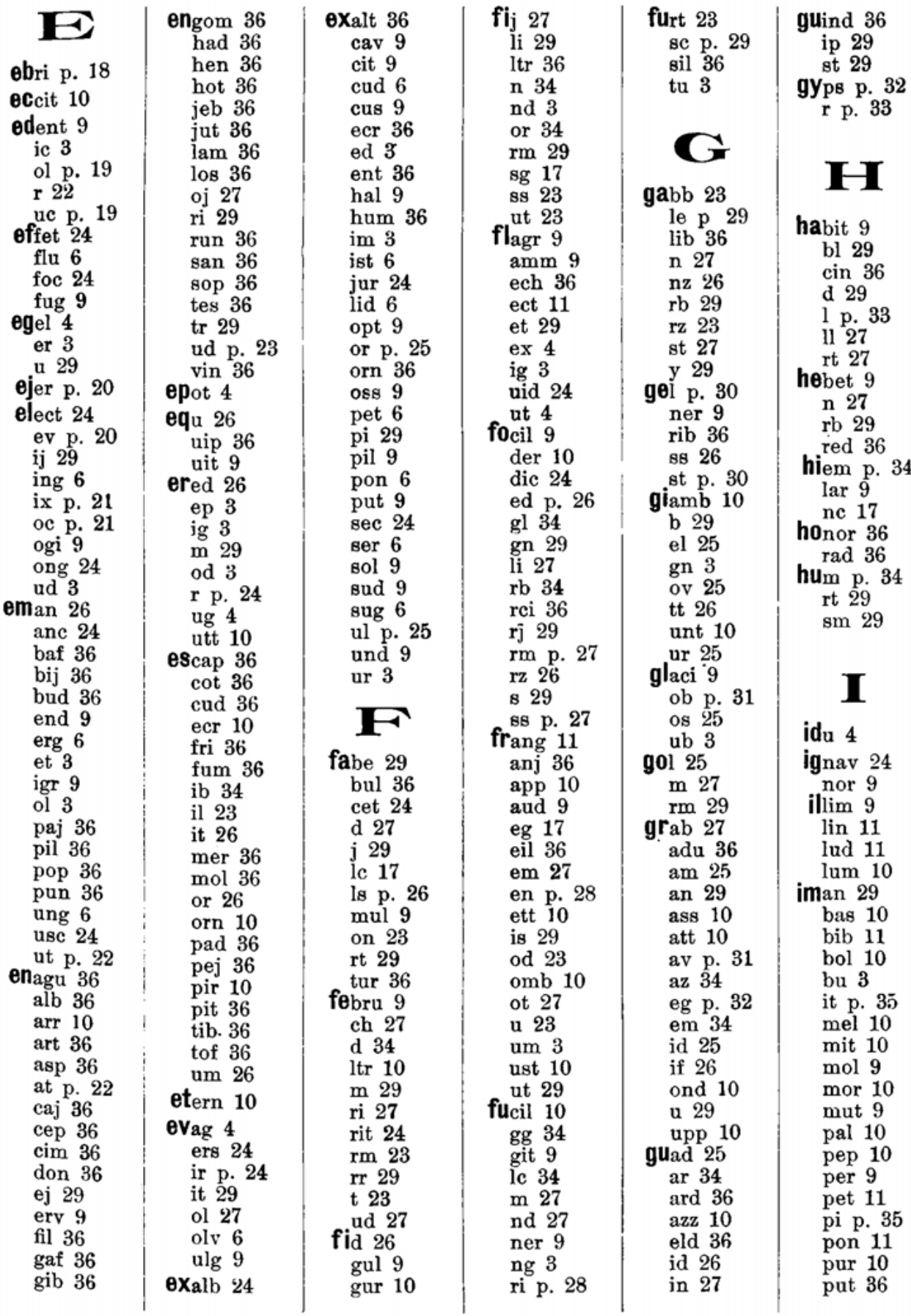




\begin{tabular}{|c|c|c|c|c|c|}
\hline 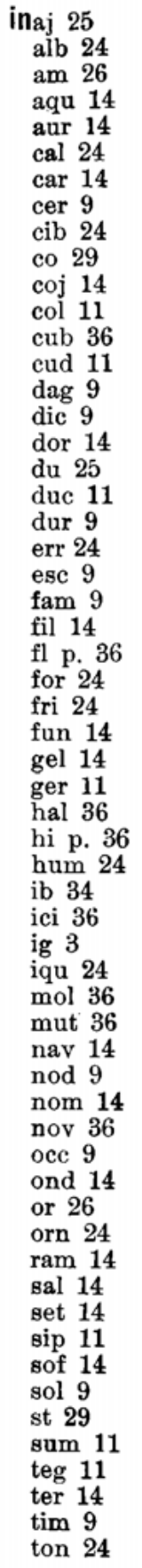 & $\begin{array}{c}\text { intr p. } 37 \\
\text { tu } 3 \\
\text { tuf } 14 \\
\text { um } 25 \\
\text { unc } 9 \\
\text { und } 36 \\
\text { ung } 11 \\
\text { ur } 3 \\
\text { vad } 11 \\
\text { vas } 14 \\
\text { ve } 34 \\
\text { veh } 11 \\
\text { vej } 36 \\
\text { vit } 9 \\
\text { voc } 9 \\
\text { vol } 14 \\
\text { iris } 27 \\
\text { rad } 11 \\
\text { rep } 11 \\
\text { ret } 14 \\
\text { rig } 9 \\
\text { rit } 36 \\
\text { ror } 14 \\
\text { ru } 3 \\
\text { ruf } 9 \\
\text { iscus } 14 \\
\text { ol } 26 \\
\text { tri } 36 \\
\text { iter p. } 37\end{array}$ & 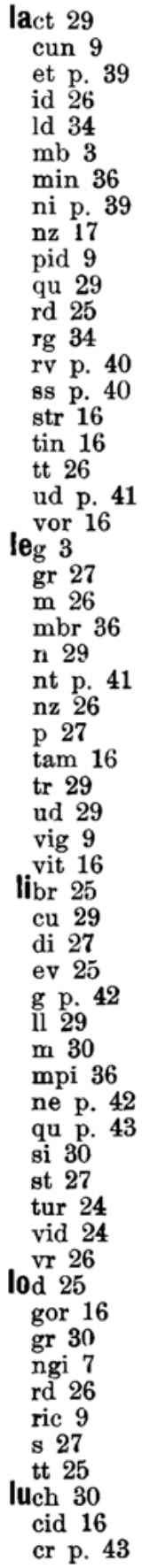 & $\begin{array}{l}\text { macer } 9 \\
\text { ci } 4 \\
\text { cin } 16 \\
\text { did } 9 \\
\text { dur } 36 \\
\text { est } 7 \\
\text { gn } 26 \\
\text { go } 30 \\
\text { lax } 9 \\
\text { lt } 34 \\
\text { lus } 16 \\
\text { lv } 30 \\
\text { mm } 25 \\
\text { n p. } 44 \\
\text { nch } 36 \\
\text { nd } 25 \\
\text { nej } 36 \\
\text { ns } 26 \\
\text { ran } 36 \\
\text { rid } 36 \\
\text { rit } 9 \\
\text { rn } 25 \\
\text { rz } 26 \\
\text { ss } 25 \\
\text { tur } 9 \\
\text { ul } 30 \\
\text { yor } 36 \\
\text { zn } 30 \\
\text { med } 30 \\
\text { ll } 30 \\
\text { mor } 9 \\
\text { nom } 16 \\
\text { nor } 36 \\
\text { rit } 16 \\
\text { rl } 25 \\
\text { rm } 27 \\
\text { rs p. } 45 \\
\text { rt } 26 \\
\text { s } 27 \\
\text { tu } 3 \\
\text { mim } 27 \\
\text { ngu } 36 \\
\text { ni p. } 45 \\
\text { nor } 9 \\
\text { nu } 34 \\
\text { r } 4 \\
\text { rl } 27 \\
\text { rr } 25 \\
\text { sur } 16\end{array}$ & $\begin{array}{l}\text { I } \\
\text { narr p. } 46 \\
\text { s } 26 \\
\text { sc } 3 \\
\text { sp } 25 \\
\text { tur } 16 \\
\text { v p. } 47 \\
\text { nec p. } 47 \\
\text { ct } 3 \\
\text { gum } 7 \\
\text { rb } 25 \\
\text { rvi } 36 \\
\text { tt } 25 \\
\text { niel } 30 \\
\text { ell } 16 \\
\text { gr p. } 48 \\
\text { tid } 7 \\
\text { nod p. } 48 \\
\text { dr } 34 \\
\text { j } 26 \\
\text { min } 16 \\
\text { t } 25 \\
\text { vel } 36 \\
\text { ver } 16 \\
\text { nub } 3 \\
\text { bl } 27 \\
\text { d } 25 \\
\text { mer } 9\end{array}$ & 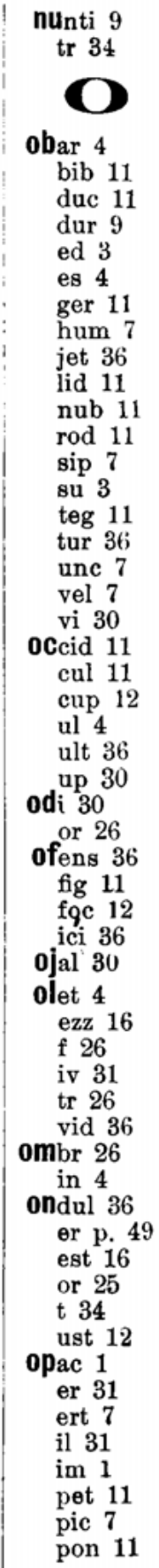 \\
\hline
\end{tabular}




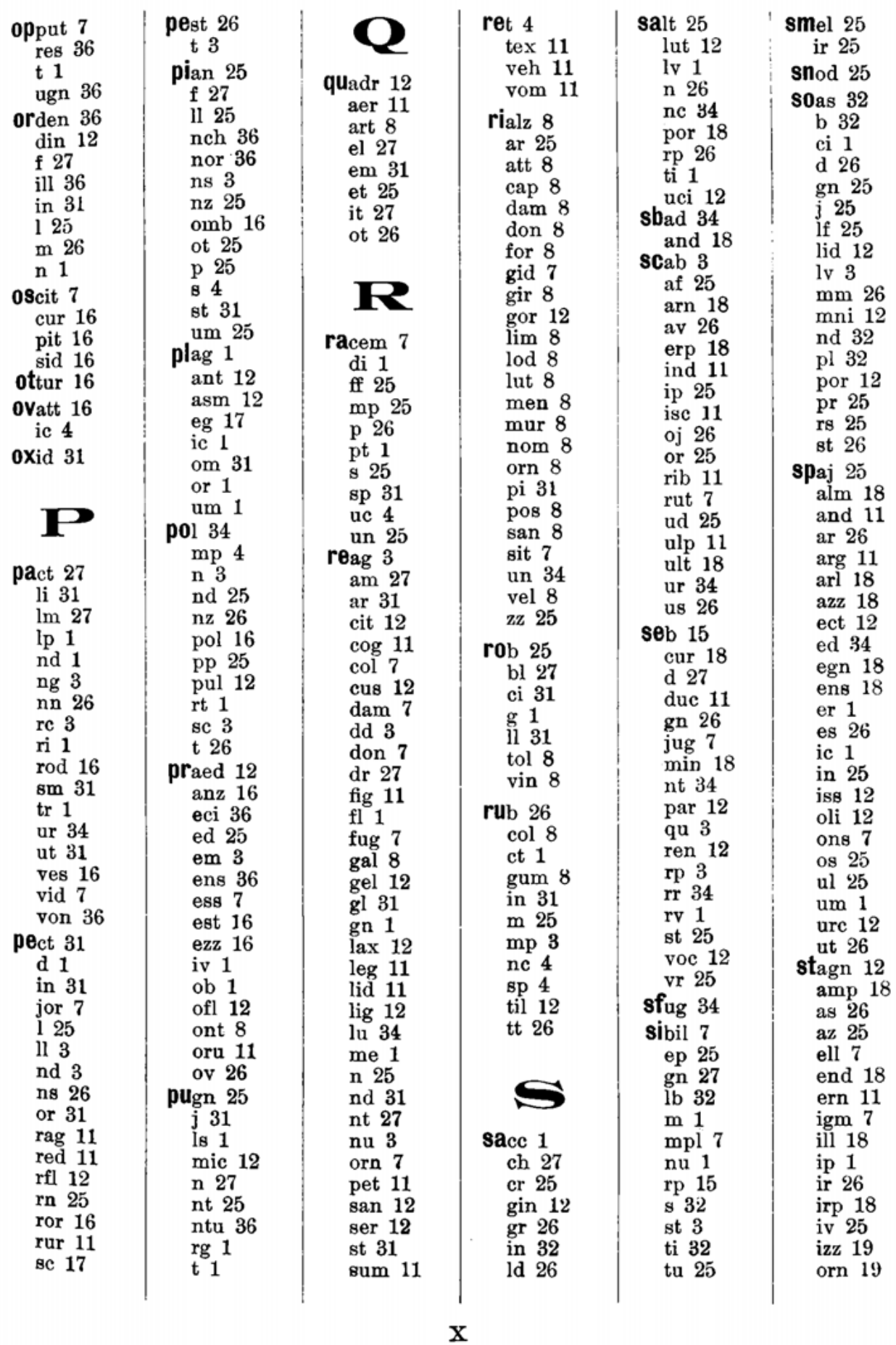




\begin{tabular}{|c|c|c|c|c|c|}
\hline $\begin{array}{l}\text { strem } 19 \\
\text { rep } 11 \\
\text { ru } 34 \\
\text { uf } 26 \\
\text { upr } 12 \\
\text { urb } 19 \\
\text { ut } 25 \\
\text { subar } 7 \\
\text { big } 11 \\
\text { blu } 11 \\
\text { bur } 11 \\
\text { cid } 7 \\
\text { d } 1 \\
\text { ffl } 12 \\
\text { fl } 32 \\
\text { gil } 12 \\
\text { lc } 1 \\
\text { mm } 15 \\
\text { on } 25 \\
\text { pin } 12 \\
\text { pp } 15 \\
\text { zz } 26 \\
\\
\text { T } 1 \\
\text { tabul } 7 \\
\text { cit } 19\end{array}$ & $\begin{array}{l}\text { tali } 1 \\
\text { min } 7 \\
\text { p } 32 \\
\text { rd } 1 \\
\text { rj } 32 \\
\text { rp } 25 \\
\text { ss } 26 \\
\text { st } 25 \\
\text { x } 1 \\
\text { tech } 32 \\
\text { mer } 12 \\
\text { mpr } 19 \\
\text { mpt } 12 \\
\text { nt } 25 \\
\text { nu } 1 \\
\text { pid } 7 \\
\text { rs } 32 \\
\text { rti } 12 \\
\text { rz } 25 \\
\text { sor } 19 \\
\text { st } 27 \\
\text { tr } 15 \\
\text { tild } 27 \\
\text { nt } 27 \\
\text { r } 32 \\
\text { tol } 19 \\
\text { zn } 32\end{array}$ & $\begin{array}{l}\text { told } 33 \\
\text { ler } 12 \\
\text { m } 33 \\
\text { nd } 26 \\
\text { pp } 26 \\
\text { rn } 33 \\
\text { rp } 1 \\
\text { rt } 34 \\
\text { s } 25 \\
\text { trab } 33 \\
\text { act } 12 \\
\text { ad } 34 \\
\text { an } 1 \\
\text { at } 27 \\
\text { em } 26 \\
\text { ep } 33 \\
\text { in } 26 \\
\text { ipl } 7 \\
\text { it } 25 \\
\text { ot } 26 \\
\text { ov } 25 \\
\text { uf } 33 \\
\text { us } 15 \\
\text { tuff } 25 \\
\text { mb } 33 \\
\text { mul } 12 \\
\text { nic } 7\end{array}$ & $\begin{array}{c}\text { T } \\
\text { uber } 1 \\
\text { ulcer } 12 \\
\text { ul } 1 \\
\text {-uman } 25 \\
\text { br } 1 \\
\text { ect } 12 \\
\text { ett } 19 \\
\text { uncin } 19 \\
\text { izz } 19 \\
\text { urin } 15 \\
\text { t } 26 \\
\text { ustil } 12 \\
\text { urp } 12 \\
\text { util } 25 \\
\text { uvi } 33 \\
\\
\text { vaci } 33 \\
\text { cu } 1\end{array}$ & $\begin{array}{l}\text { vall } 1 \\
\text { lor } 19 \\
\text { lu } 33 \\
\text { lut } 19 \\
\text { nt } 25 \\
\text { por } 12 \\
\text { r } 25 \\
\text { st } 1 \\
\text { vect } 1 \\
\text { get } 12 \\
\text { len } 19 \\
\text { lit } 7 \\
\text { nd } 27 \\
\text { nen } 12 \\
\text { nt } 26 \\
\text { rs } 26 \\
\text { ss } 25 \\
\text { tu } 15 \\
\text { x } 1 \\
\text { vibr } 1 \\
\text { ci } 27 \\
\text { dim } 19 \\
\text { et } 25 \\
\text { gi } 33 \\
\text { gil } 12 \\
\text { gn } 26 \\
\text { lt } 27\end{array}$ & $\begin{array}{c}\text { zaf } 27 \\
\text { ff } 26 \\
\text { mp } 26 \\
\text { nj } 33 \\
\text { rp } 33 \\
\text { zel } 26 \\
\text { pp } 25 \\
\text { zinn } 25 \\
\text { zomb } 25 \\
\text { n } 25 \\
\text { zurr } 33\end{array}$ \\
\hline
\end{tabular}


